\title{
Monitorización de instrumentación avanzada para docencia en red.
}

Juan Bta. Talens, José Pelegrí-Sebastià, Fco José Ibáñez y Tomás Sogorb.

Universitat Politècnica de Valéncia, jpelegri@upv.es.

\begin{abstract}
Due to university internationalization, learning's concept is not linked to a specific geographical place. This document describes the methods used to visualize the laboratory's instruments (oscilloscope Tektronix TDS 210 and the generator Agilent 33120A) monitored remotely. Being able to generalize to any instrument that has an interface for remote connection regardless of the type of bus technology.
\end{abstract}

Keywords: LabVIEW, Osciloscope, Tektronix, Function Generator, Agilent.

\begin{abstract}
Resumen
Debido a la internacionalización universitaria, el concepto aprendizaje no está vinculado a un lugar geográfico específico. Este documento describe los métodos utilizados para visualizar en el laboratorio los instrumentos (osciloscopio Tektronix TDS 210 y el generador Agilent 33120A) monitorizados de forma remota. Pudiéndose generalizar a cualquier instrumento que tenga interfaz para conexión remota independientemente del tipo de tecnología bus.
\end{abstract}

Palabras clave: LabVIEW, Osciloscopio, Tektronix, Generador de funciones, Agilent.

\section{Introducción}

La asignatura de Instrumentación Avanzada estudia con detalle diferentes instrumentos básicos en un laboratorio de telecomunicaciones y el control de estos a través de diferentes buses de instrumentación (GPIB, VXI, Ethernet, USB). Estos contenidos cubren un amplio rango de conceptos prácticos de un instrumento convencional y las herramientas que hacen posible realizar un instrumento virtual y su control. El internet de las cosas nos brinda la oportunidad de convertir algunas computadoras alrededor del mundo en sofisticados laboratorios (Yang-Mei and Bo, 2014) o donde simplemente un alumno o alumna pueda hacer sus prácticas como p. ej. (Amer et al., 2015), (Selangor, 2017) y (Kamensky et al., 2017). El presente artículo detalla el diseño y desarrollo de una aplicación capaz de mostrar en pantalla el estado de los instrumentos del laboratorio de electrónica. En concreto el osciloscopio y el generador de funciones, elementos básicos en la realización de prácticas con LabVIEW. Esta aplicación surge con la imposibilidad de algunos alumnos acceder al laboratorio por encontrarse ocupado o por incompatibilidad de horarios. Así pues, se recogen las observaciones de los alumnos que han cursado la asignatura desde el curso 
académico 2012-2013 hasta el curso 2016-2017 y que han tenido la posibilidad de utilizarla.

\section{Objetivo}

El objetivo de la monitorización de instrumentación en red es favorecer el acceso remoto al laboratorio de los alumnos que por incompatibilidad de horarios o por encontrarse desplazados les es imposible acceder físicamente.

\section{Desarrollo de la innovación}

\subsection{Especificaciones}

Se requiere el diseño y desarrollo de una aplicación capaz de mostrar en pantalla el estado de los instrumentos del laboratorio de electrónica. La aplicación debe proporcionar el control de errores y no debe permitir desde la misma el control WYSIWYG de los mismos p. ej. cambios de escala, señal etc. El usuario únicamente podrá controlar el instrumento mediante el uso de LabVIEW (National Instruments, 2016) u otro software. Por tanto, la aplicación únicamente debe mostrar el estado de los mismos.

Se establecen tres condiciones:

- El alumno debe acceder respetando un determinado control horario de acceso al equipo informático y la sesión activa tiene un máximo de dos horas. Si el alumno deja inactiva la sesión se debe desconectar automáticamente a los 10 minutos. Si otro usuario alumno, accede fuera de su horario marcado podrá acceder si la sesión está libre. Por el contrario, si está siendo usada la sesión, el sistema pedirá permiso al usuario activo para permitir el acceso o denegarlo.

- Para el control y evaluación del profesor, se requiere de registro de control de acceso a los equipos, en que se pueda realizar una auditoría del usuario que se conecta, la hora de inicio y la hora de finalización.

- Para que el sistema sea sostenible desde la perspectiva económica y medioambiental los equipos tanto informáticos como instrumental se deben enchufar en el horario de trabajo, quedando totalmente apagados fuera de ese horario.

\subsection{Tecnologías utilizadas}

\subsubsection{Condiciones iniciales del entorno}

Se dispone de un laboratorio con equipos informáticos en un entorno WINDOWS, versión para empresas (WINDOWS 7 ENTERPRISE ED., WINDOWS 10, etc.) conectados en red y unidos a un dominio (GANDIELAB2008) de ACTIVE DIRECTORY bajo WINDOWS 2008 SERVER o posterior. En cada puesto de trabajo hay un equipo informático y un osciloscopio digital Tektronix TDS210 y un generador de funciones HP33120A ambos 
controlados por GPIB. Además, dispone de una fuente de alimentación regulable y cortocircuitable Promax FA-662B y otras herramientas y componentes.

\subsubsection{Escritorio remoto}

Escritorio remoto (Meneses Wong, 2008) conecta dos equipos a través de una red o de Internet. Una vez establecida la conexión, se observa el escritorio del PC remoto como si se estuviese sentado frente a él, y se tiene acceso a todos sus programas y archivos. Dado que los equipos del laboratorio de electrónica poseen el S.O. WINDOWS y que esta función está incluida en todas las ediciones. Esta tecnología ofrece de una forma rápida y sencilla una solución a la conectividad entre equipos remotos.

\subsubsection{LabVIEW}

Es una plataforma propietaria para programación a gran escala que permite el desarrollo de aplicaciones generalmente orientadas al control de la Instrumentación, además de estar presente con una carga alta en los contenidos de las asignaturas de Instrumentación Avanzada de la titulación Grado en Ingeniería de Telecomunicación, Sonido e imagen de la Escuela Politécnica Superior de Gandia.

\subsubsection{MAX (Measurement \& Automation Explorer)}

Measurement \& Automation Explorer (National Instruments, 2016) proporciona acceso a los instrumentos conectados a los periféricos de la computadora, permitiendo su monitorización y detección de fallos. Aunque ofrece amplias posibilidades el uso de este software se ha limitado a la depuración de errores en el análisis de etapas tempranas de diseño.

\subsubsection{GPIB}

El Hewlett-Packard Instrument Bus (HP-IB) es un estándar bus de datos digital de corto rango desarrollado por Hewlett-Packard en los años 1970 para conectar dispositivos de test y medida p. ej. multímetros, osciloscopios, etc, con dispositivos que los controlen como un ordenador. Otros fabricantes copiaron el HP-IB, llamando a su implementación General Purpose Instrumentation Bus (GPIB). En 1978 el bus fue estandarizado por el Institute of Electrical and Electronics Engineers (IEEE) como el IEEE-488 (488.1).

El IEEE-488 permite que hasta 15 dispositivos inteligentes compartan un simple bus paralelo de 8 bits, mediante conexión en cadena, con el dispositivo más lento determinando la velocidad de transferencia. La máxima velocidad de transmisión está sobre 1 Mbps en el estándar original y en 8 Mbps con IEEE-488.1-2003 (HS-488).

\subsection{Soluciones}

\subsubsection{LabVIEW}

La captura de imagen mediante el uso del comando HARDCopy proporciona la ventaja que no se deben obtener otros parámetros y traducirlos a un lenguaje visual para su 
interpretación por el usuario. Este hecho hace que se descarte la opción de copiar los puntos ya que implica el envío de 10 MS y además las cabeceras de toda la configuración que llevan a la saturación del bus. Por otra parte, la implementación al lenguaje visual requiere de una elevada complejidad en la aplicación.

\subsubsection{Elección del BUS}

El uso de dos buses, soportado en el caso del osciloscopio, nace en previsión que el usuario reciba constantemente actualizaciones de la imagen. Se comprueba que esto es inviable ya que el bus se bloquea y por lo tanto no es posible enviar ni recibir comandos. Por lo que se decidió restringir la captura a petición del usuario. Este sólo recibe cuando pulsa el botón actualizar. No obstante, la idea del uso del RS232 para la captura de la imagen sigue siendo interesante porque permite mayor velocidad que el GPIB. Se puede configurar el osciloscopio hasta 19200 baudios, aunque requiere de una configuración previa del instrumento que pudiera no ser compatible con el reseteo del mismo e inducir al usuario a errores de interpretación, o en el peor de los casos, a imposibilitar la conexión.

\subsubsection{Control de errores}

La monitorización por parte del IOtrace se descarta ya que los errores que muestra no están codificados como en los manuales de los instrumentos por lo que sacar del mismo información para después mostrarla en la aplicación supone una carga computacional adicional que ejecutar el MAX en la misma máquina. Se decide incorporar en la aplicación la información de errores a petición del usuario

\subsubsection{Configuración del sistema}

Usuarios y grupos: Desde la consola de Active Directory Users and Computers del dominio informático WINDOWS, en nuestro caso GANDIELAB2008, se crea un Grupo de Seguridad de tipo Dominio Local, GrupoEscritorioRemotoIns. También se crean tantos usuarios como alumnos deben conectarse por Escritorio Remoto. Cada usuario debe ser miembro del grupo.

El equipo del laboratorio debe tener activado el Servicio de Escritorio Remoto desde la configuración del sistema, apartado Escritorio Remoto. Además, se debe agregar el grupo de dominio "GrupoEscritorioRemotoIns" desde la consola de Administración de Equipo (local) al grupo "Usuarios de Escritorio Remoto" ubicado en Herramientas del sistema > Usuarios y Grupos Locales > Grupos

Igualmente se deben revisar las reglas del firewall y del antivirus para verificar que está permitida la conexión externa desde Escritorio Remoto.

Tiempos: Para el control tiempos debemos ir a la pestaña sesiones dentro de las propiedades del usuario tal como vemos en la figura 1. 


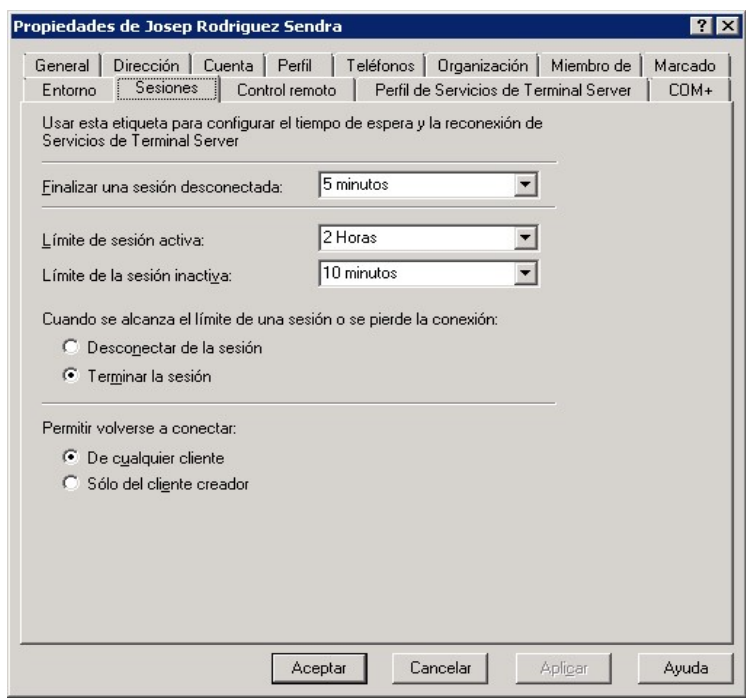

Figura 1. Datos para el control de tiempo.

Auditoría: Para abordar la segunda condición correspondiente al registro de actividad y acceso al equipo se realiza un doble sistema de auditoría. Una activando la auditoría del sistema operativo y la otra desde un fichero de texto accesible a través de red que refleje inicio o cierre, nombre de equipo, usuario fecha y hora.

Para la primera auditoría se activa desde la directiva de grupo de controlador de dominio (GPO) que el sistema refleje automáticamente en los Registros del Sistema. Estos son accesibles desde la consola del Visor de Sucesos del controlador de dominio como los siguientes eventos:

- Auditar eventos de inicio de sesión

- Auditar eventos de inicio de sesión de cuenta

Con esta auditoría queda almacenada una gran cantidad de información respecto a los inicios y cierres de sesión de usuario. Esta información es demasiado pesada de manejar para los fines docentes que se persiguen y por ello, se establece la segunda forma de registro de actividad que es más sencilla, quedando la primera con fines de resolución de conflictos y redundancia.

La segunda forma de registro de actividad y acceso consta de unos Scripts que se ejecutan en el equipo de laboratorio al inicio y cierre de sesión de usuario remoto y que escriben una línea de texto con la siguiente información para cada evento de inicio o cierre:

Inicio, nombre equipo, usuario, fecha y hora tal y como puede verse en la figura 2. 


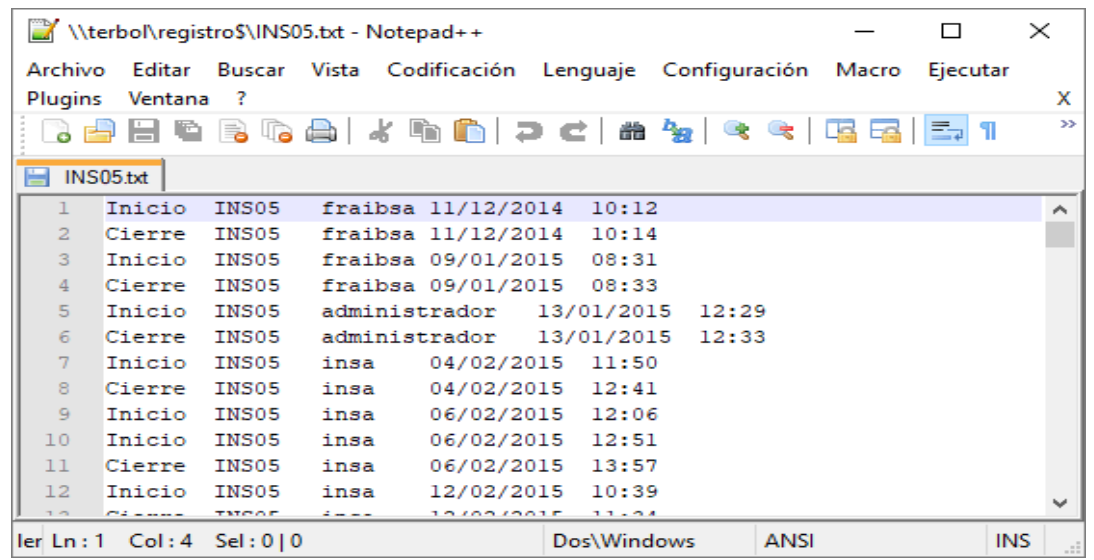

Figura 2. Registro de actividad.

Para implementar este registro se debe generar un recurso compartido oculto en el controlador de dominio, p. ej. Iterbollregistro\$; . Los administradores de sistema y el profesorado deben tener permisos de acceso. En esta carpeta se crean dos ficheros de texto vacíos con el nombre de los equipos informáticos que se pretende registrar. Por ejemplo INS05.txt y INS06.txt y se les asigna permisos de "escritura" para el usuario "todos".

También requiere dejar en este recurso los scripts que deberemos lanzar al inicio y cierre de sesión para registrar los accesos; inicio.cmd, cierre.cmd y control_insa.cmd.

\section{- Inicio.cmd}

Este script será lanzado al inicio de la sesión y llamará al control_insa.cmd para que registre el evento. Lanza en siguiente comando:

call \\terbol\registro\$\control_insa.cmd Inicio

- $\quad$ cierre.cmd

Este script se lanza al cierre de la sesión

call \} \backslash \text { terbol\registro\$\control_insa.cmd Cierre }

- control_insa.cmd

Este es el script principal que permite escribir cada una de las filas del fichero de texto con la información solicitada y lo realiza de la siguiente manera:

echo off

set dia=\%DATE\%

FOR /F "tokens=*" \%\%A IN ('TIME/T') DO SET hora=\%\%A

if \%computername\%==INS05 echo \%1 \%computername\% \%username\%

$\%$ dia\%

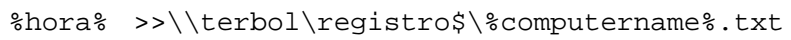

if \%computername\%==INS06 echo \%1 \%computername\% \%username\% \%dia\%

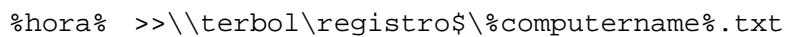

2018, Universitat Politècnica de València

Congreso IN-RED (2018) 
Para mejorar su privacidad, es interesante que estos ficheros se les active el atributo de "ocultos". Para lanzar estos scripts se requiere de una directiva GPO del dominio. Para ello, a través de la consola de "Administración de Directivas de Grupo" del dominio WINDOWS, se activan las políticas de ejecución de scripts requeridas tal y como podemos ver en la figura 3. En dicha consola se crea un GPO en dicho dominio i.e. "Auditoria acceso inicio-cierre sesión" y se vincula. En la ruta "Configuración de usuario > directivas > Configuración de Windows > Scripts" se requiere agregar para el elemento "Iniciar sesión" el fichero "inicio.cmd" y para el elemento "Cerrar sesión" el fichero "cierre.cmd".

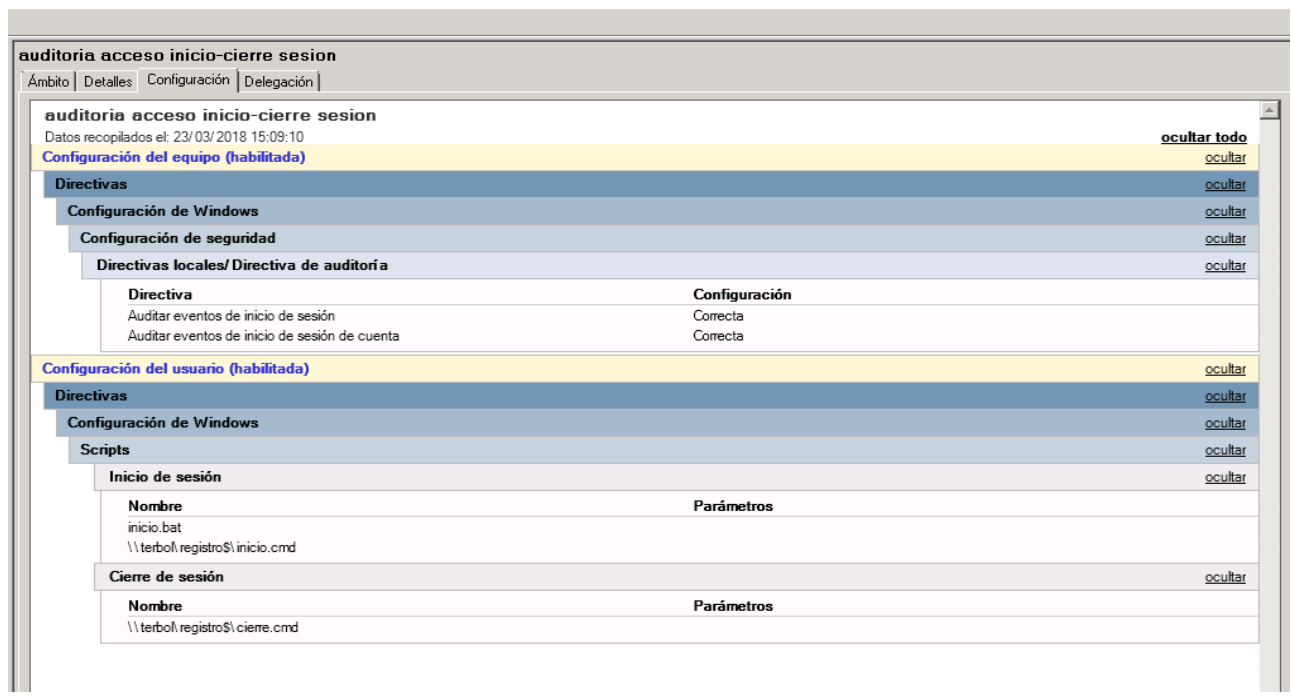

Figura 3. Configuración de políticas de ejecución.

A cada usuario se les proporciona un archivo ejecutable para realizar la conexión.

\section{Contenido del archivo para la conexión:}

ConexinER(pass=insa).rdp

screen mode id:i:2 use multimon:i:0 desktopwidth:i:800 desktopheight:i:600 session bpp:i:32

winposstr:s:0,3,0,0,800,600 compression:i:1 keyboardhook:i:2 audiocapturemode:i:0 videoplaybackmode:i:1 connection type:i:7 networkautodetect:i:1 bandwidthautodetect:i:1 displayconnectionbar:i:1 username:s:gandielab2008linsa4 enableworkspacereconnect:i:0 disable wallpaper:i:0

allow font smoothing:i:0 allow desktop composition:i:0 disable full window drag:i:1 disable menu anims:i:1 disable themes:i:0

disable cursor setting:i:0 bitmapcachepersistenable:i:1 full address:s:ins06.gnd.upv.es audiomode:i:0 redirectprinters:i:1 redirectcomports:i:0 redirectsmartcards:i:1 redirectclipboard:i:1 redirectposdevices:i:0 autoreconnection enabled:i:1 authentication level:i:2

prompt for credentials:i:0 negotiate security layer:i:1 remoteapplicationmode:i:0 alternate shell:s:

shell working directory:s: gatewayhostname:s: gatewayusagemethod:i:4 gatewaycredentialssource:i:4 gatewayprofileusagemethod:i:0 promptcredentialonce:i:0 gatewaybrokeringtype:i:0

use redirection server name:i:0 rdgiskdcproxy:i:0 kdcproxyname:s:

(cc)) EY-NC-ND 2018, Universitat Politècnica de València

Congreso In-Red (2018) 


\subsubsection{Sostenibilidad}

Para el ahorro energético y sostenibilidad se ha configurado la BIOS del equipo informático de tal manera que se despierte automáticamente a una determinada hora usando el "Wake Up" de las opciones de la pestaña "Power Management”. Para el apagado del equipo se utiliza las funciones de ahorro de energía propias del sistema operativo local. Mediante el uso de un controlador horario programable Orbis Tempo+ como el de la figura se optimiza el control del consumo energético.

\subsection{Detalles de implementación}

La implementación del sistema en LabVIEW se desarrolla por separado para luego unirse en un proyecto. Como puede verse en la figura 4 la aplicación consiste en dos subprogramas, uno para el osciloscopio y otro para el generador de señales. Tanto el osciloscopio como el generador realizan dos procesos, captura de la imagen y captura de errores. El generador dependiendo de que haya seleccionado el usuario únicamente muestra dicha selección. Por lo que los subprocesos de este último, como son error, frecuencia, amplitud, offset y forma de onda derivan de una única petición al instrumento.

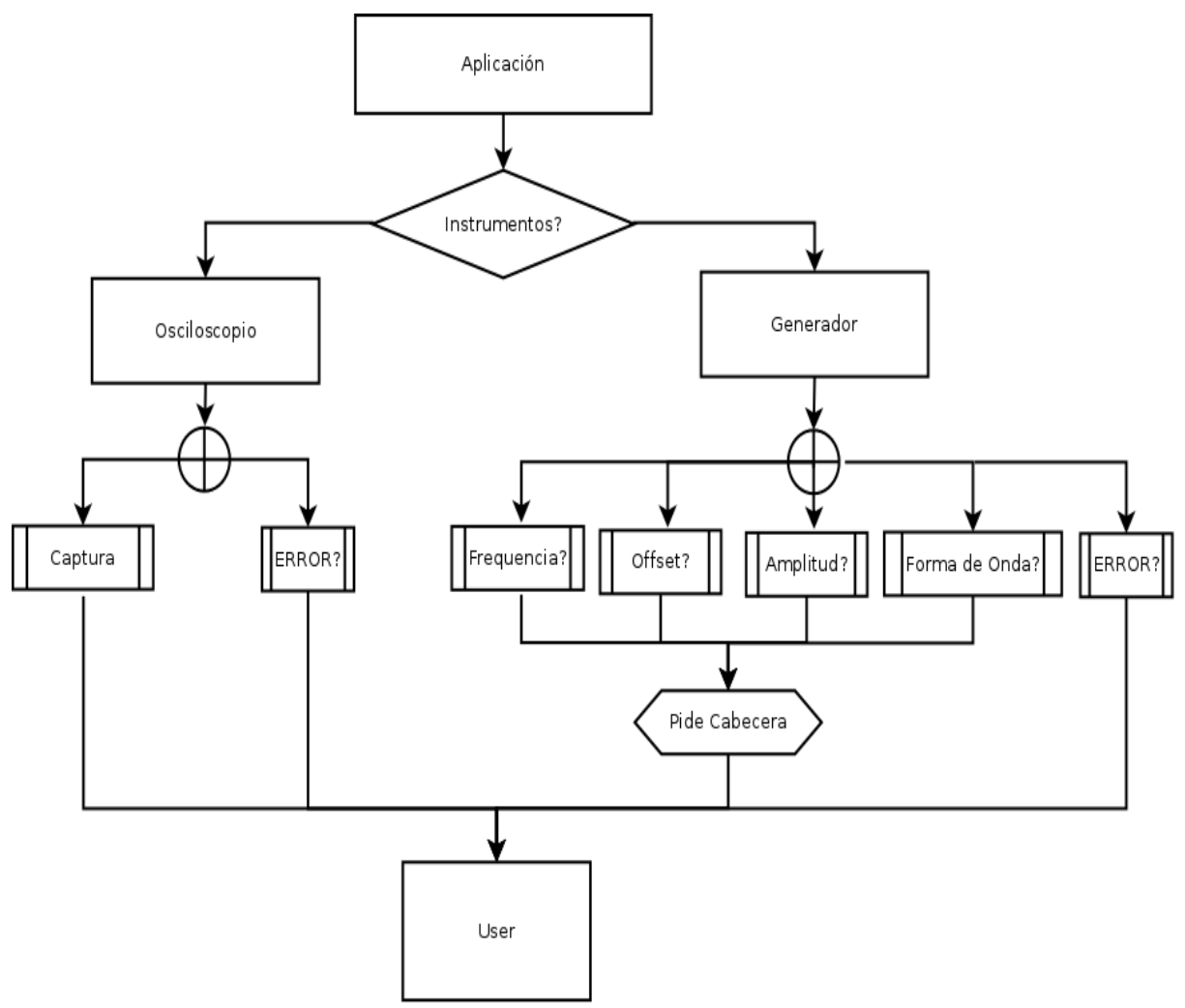

Figura 4. Diagrama de flujo de la aplicación.

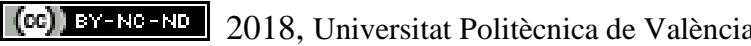

Congreso IN-RED (2018) 


\subsubsection{Búsqueda de Instrumentos}

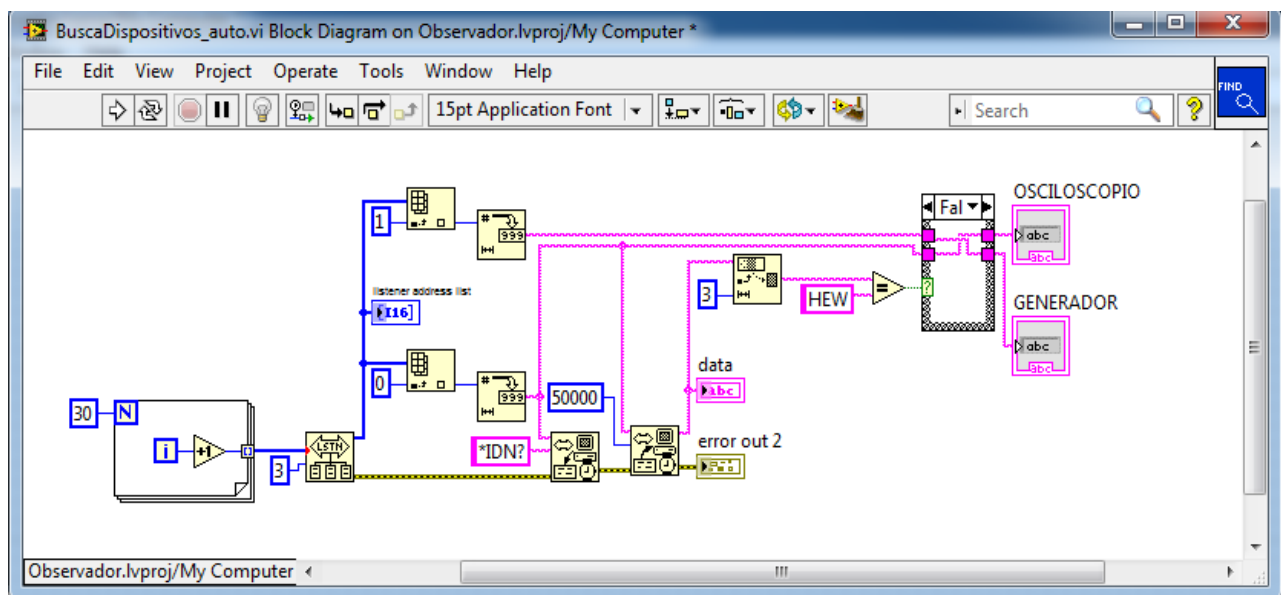

Figura 5. Diagrama de Bloques BuscaDispositivos auto.vi

Para que la aplicación sea universal a todos los PC's del laboratorio se incluye un *.vi que se encarga de buscar los dispositivos e identificarlos para su uso en los otros *.vi's. En la figura 5 se puede observar el diagrama de bloques del código. Si se observa el código se intuye como se está pidiendo que se identifiquen los instrumentos y se compara con las tres primeras letras que corresponden a la denominación del generador de funciones 'HEWLETT-PACKARD,33120A,0,10.0-5.0- 1.0'. Una vez identificado o descartado el generador resulta trivial asignar a los indicadores correspondientes el valor de la dirección del instrumento

\subsubsection{Osciloscopio}

El osciloscopio sigue dos secuencias. Inicialmente se encuentra el instrumento y se crea un archivo *.bmp en la carpeta de recursos temporales. Se registra un path que utilizara el *.vi para convertir los datos en imagen. En la segunda secuencia previa pulsación del botón “Actualizar” se envían los comandos vistos en la figura 6 y se bloquea el cursor hasta que se capturan los datos. Con los datos se genera la imagen que se envía al visor. El *.vi reacciona a petición del usuario mediante la captura de eventos. Los eventos posibles dependen de los tres botones que existen; Error, Actualizar y ON/OFF.

(c) EY-NC-ND 2018, Universitat Politècnica de València 


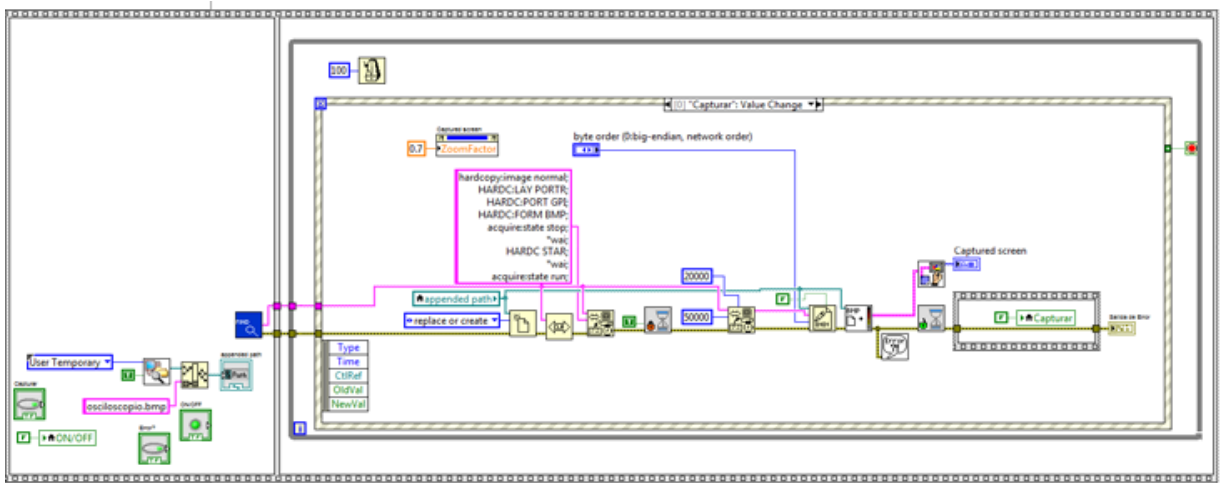

Figura 6. Diagrama de bloques archivo Osciloscopio.vi.

\subsubsection{Generador de funciones}

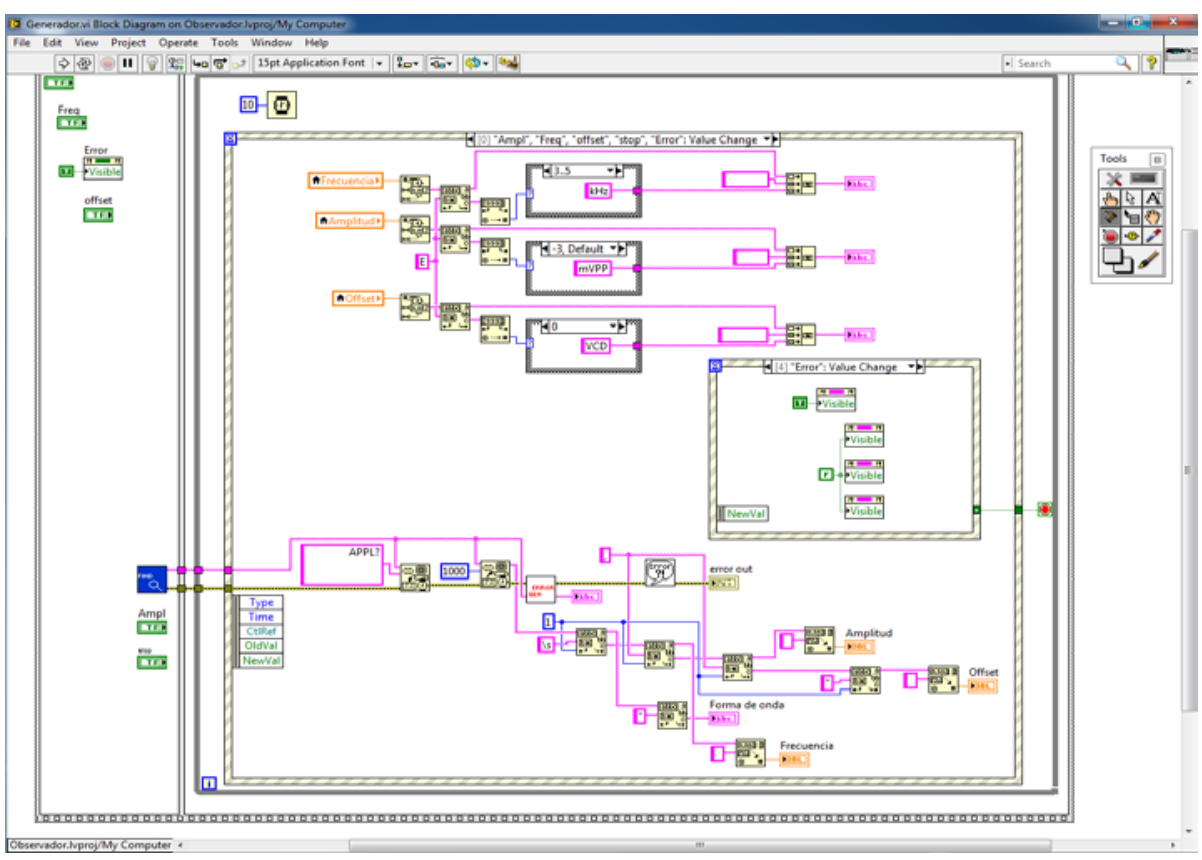

Figura 7. Diagrama de bloques archivo Generador.vi.

El generador de funciones sigue una secuencia donde en una primera fase se encuentra el dispositivo mediante el *.vi visto en $\mathrm{V}$-A y en la segunda se queda en un bucle mientras no se actúe sobre el botón de "OFF". Es en dicho bucle donde se pide al instrumento el estado del mismo mediante el comando APPL? y, dependiendo del botón pulsado se muestra el texto requerido en pantalla. En la figura 7 puede verse un detalle de la implementación.

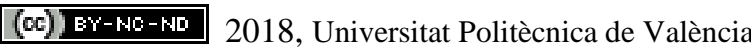

Congreso IN-RED (2018) 


\subsubsection{Errores del generador de funciones}

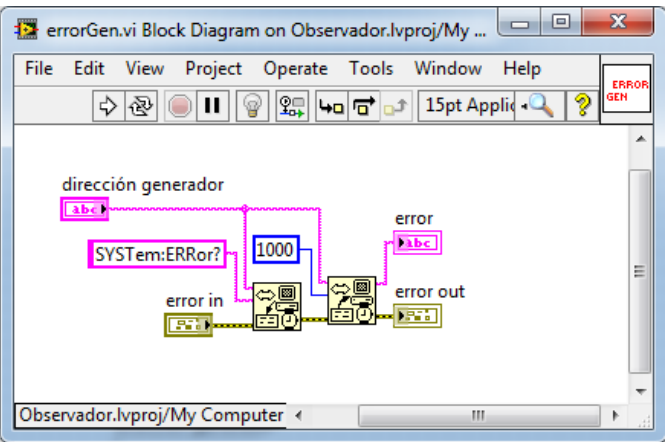

Figura 8. Diagrama de bloques archivo errorGen.vi

Tal y como se deduce del diagrama de bloques del *.vi, visto en la figura 8, se recoge la respuesta del instrumento al comando 'SYSTem:ERRor?'.

\subsubsection{Coma decimal}

En el sistema anglosajón no se utiliza la coma para indicar que empieza la mantisa de un número, en lugar de eso, se utiliza el punto. Este hecho obliga al programador a tener en cuenta un posible error de interpretación en el sistema operativo según la configuración regional establecida. LabVIEW permite tener en cuenta el uso de la coma para la separación decimal, pero en la compilación del proyecto se observó que no se disponen de la compatibilidad con el idioma español y por tanto al compilar resultó imposible establecer dicha configuración. Por lo que se optó por utilizar en el equipo destinado al uso de la aplicación la configuración anglosajona.

\subsection{INSTALACIÓN Y USO}

\subsubsection{Conexión remota}

Tras configurar la conexión VPN necesaria para acceder a equipos fuera de la Universidad Politécnica de Valencia, explicado paso a paso en (UPV- Área de Sistemas de la Información y las Comunicaciones) sección de información de la UPV. Se debe ejecutar el archivo 'Conexion ER (PASS=insa).rdp' para que realice la petición de conexión remota al equipo ins06.gnd.upv.es, el cual debe estar iniciado. Se selecciona la opción 'Usar otra cuenta', y se introduce el usuario y la contraseña proporcionada, por ejemplo, 'gandielab2008\insa4' e 'insa4'. Una vez en el escritorio remoto, se accede a la ruta donde se encuentra la aplicación y se ejecuta el acceso directo 'Observador.lnk'. Tras el inicio se debería poder visualizar en el escritorio dos ventanas como en la figura 9.

2018, Universitat Politècnica de València

Congreso In-Red (2018) 


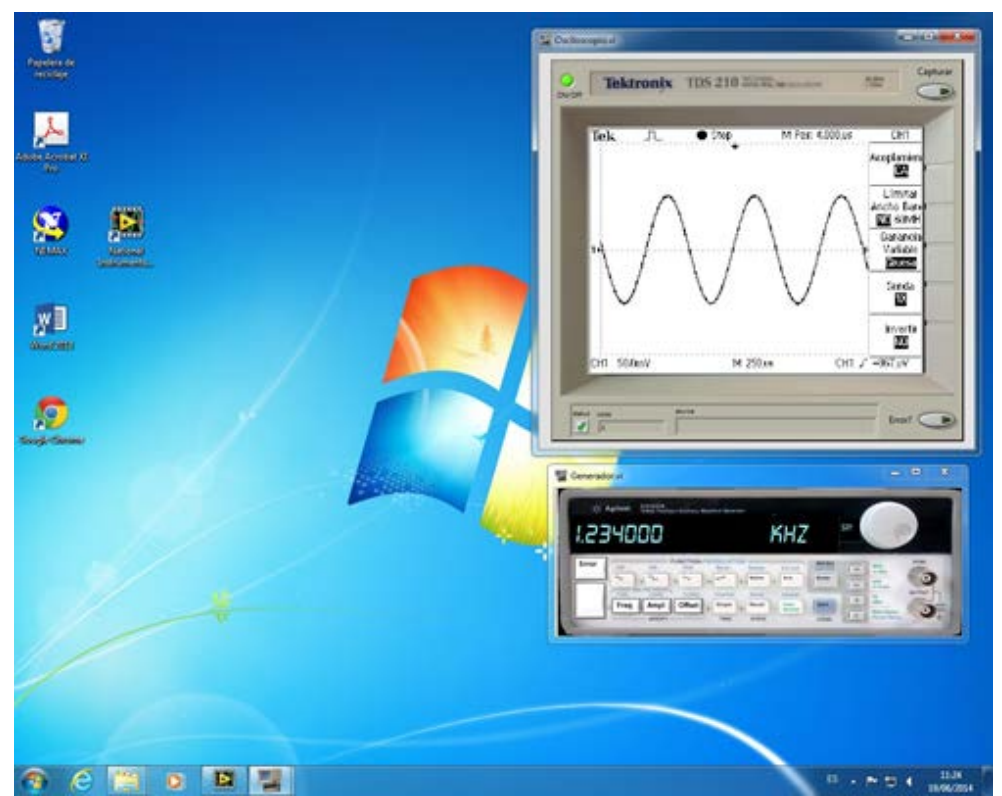

Figura 9. Captura Aplicación Observador abierta mediante Escritorio remoto.

\subsubsection{Uso del Observador}

Osciloscopio: Una vez iniciada la aplicación el usuario podrá acceder al botón de Error para visualizar si existe algún error en el dispositivo o bien al botón de Actualizar. Este último bloquea el cursor hasta que la captura se muestre en pantalla. El tiempo aproximado dependerá del equipo y la conexión siendo como mínimo de 17s.

En el caso que el instrumento no se encuentre activo o el bus esté saturado aparecerá un mensaje de error y aparecerá en la parte inferior izquierda un tick de color rojo que indica que el dispositivo no se ha encontrado y/o no se ha listado correctamente. Es posible la detención del subprograma cerrando la ventana o bien mediante el botón de ON/OFF.

Generador: Una vez iniciada la aplicación se tienen varios botones disponibles; 'Freq', 'Ampl' y 'Offset', que permiten que se muestre en el display del generador virtual los valores de frecuencia, amplitud y offset, respectivamente, que el generador real está suministrando en ese momento.

Mediante el botón 'Error' se mostrarán en pantalla los errores que haya tenido el generador al introducir los comandos u otros parámetros, en caso que los tuviera.

En el caso que el dispositivo muestre todos los valores a cero y que no existen errores o no muestre ningún valor podría significar que el instrumento está apagado o el bus saturado o desconectado, no obstante, debería aparecer un mensaje del sistema indicando el suceso. Se recomienda consultar el horario de funcionamiento del sistema. Se requiere reiniciar la aplicación con los instrumentos encendidos. Es posible la detención del subprograma cerrando la ventana o bien mediante el botón de ON/OFF. 


\section{Resultados}

La media de alumnado que ha cursado la asignatura desde la implantación del sistema descrito, en el curso escolar 2012-2013 hasta el curso 2016-2017, es de 8.2. El porcentaje de uso se mantuvo los tres primeros cursos entorno un 15\%. La disponibilidad del laboratorio junto con escasez de matrícula; 6, 7 y 6 matriculados, facilitó una atención más personalizada y propició la realización íntegra de las prácticas en el mismo sin que hubiese la necesidad imperativa de realizarlas en remoto.

En el curso académico 2015-2016 la tasa de uso alcanzó en 100\% ya que se determinó una práctica obligatoria mediante el uso de la misma. Se realizó un cuestionario donde se recoge el grado de satisfacción del alumnado con los siguientes resultados.

Tabla 1. Resultados grado de satisfacción.

\begin{tabular}{|l|c|c|c|c|c|}
\hline Curso académico & $2012-2013$ & $2013-2014$ & $2014-2015$ & $2015-2016$ & $2016-2017$ \\
\hline$N^{\circ}$ Matriculados. & 6 & 7 & 6 & 12 & 10 \\
\hline Tasa de uso de la plataforma. & $16.67 \%$ & $14.29 \%$ & $16.67 \%$ & $100 \%$ & $100 \%$ \\
\hline Acceso a la aplicación. & 5 & 5 & 5 & 4 & 4 \\
\hline Dificultad de instalación y uso. & 5 & 5 & 5 & 5 & 5 \\
\hline Horario de disponibilidad y uso. & 5 & 5 & 5 & 5 & 5 \\
\hline Aplicabilidad a la asignatura. & 5 & 3 & 5 & 5 & 5 \\
\hline Aplicabilidad a la carrera. & 5 & 3 & 4 & 5 & 5 \\
\hline
\end{tabular}

\section{Conclusiones}

El grado de utilización ha sido forzado para que tuviese un efecto considerable y se pudiese evaluar la aplicación. No obstante, la aplicabilidad y su utilidad resultan satisfactorias para el $100 \%$ de los usuarios y en entornos semipresenciales o a distancia podría ser de utilidad.

Algunas de las sugerencias responden a la activación de los equipos que por mantenimiento podrían quedar desconectados de la red y precisan de la intervención del técnico del laboratorio. Una mejora que se prevé es introducir un circuito que active un relé cuando el ordenador arranque, tomando la señal de arranque desde cualquiera de los conectores de la fuente de alimentación ATX del equipo informático o del USB. Así los instrumentos sólo estarán encendidos el tiempo que el ordenador esté activo. 


\section{Referencias bibliográficas.}

AMER, M.A., CORTINA-PUIG, M., MARTÍNEZ, V., CRUZ, J., AND MORRAL, J. (2015). "Implementation of a LabVIEW-based virtual laboratory". 2015 IEEE International Conference on Industrial Technology (ICIT), Seville, 2015, pp. 3274-3277.

JOHARI, J., AHMAD, A., MADZHI, N. K., BUNIYAMIN, N. AND KASSIM, R. A., "LabVIEW as an effective tool for problem-based learning in undergraduate engineering education," 2017 IEEE 9th International Conference on Engineering Education (ICEED), Kanazawa, 2017, pp. 169-173.

KAMENSKÝ, M., KRÁlIKOVÁ, E., ČERVEŇOVÁ, J. AND KOVÁČ, K., "Interfacing of measuring devices in educational software system for remote access," 2017 11th International Conference on Measurement, Smolenice, 2017, pp. 175-178.

MENESES WONG, L.E. Administración de escritorio remoto. Comuniquiti, 2008, pp. 1-8.

NATIONAL INSTRUMENTS (2016). LabVIEW System Design Software.

SELANGOR, S.A. (2017). LabVIEW as an Effective Tool for Problem-Based Learning in Undergraduate Engineering Education. 169-173.

UNIVERSITAT POLITÉCNICA DE VALENCIA, Área de Sistemas de la Información y las Comunicaciones "Infoacceso: Upv".

YANG-MEI, L. AND BO, C. "Electronic Circuit Virtual Laboratory Based on LabVIEW and Multisim," 2014 7th International Conference on Intelligent Computation Technology and Automation, Changsha, 2014, pp. 222-225. 